'Escuela de Psicología, Universidad de los Andes. Santiago, Chile.

${ }^{2}$ Facultad de Educación,

Pontificia Universidad

Católica de Chile. Santiago, Chile.

apsicólogo. ${ }^{\mathrm{b}}$ Biólogo.

Estudio financiado por el Fondo de Ayuda a la Investigación (FAI), proyecto No PSI-002-08, de la Universidad de los Andes, Santiago-Chile.

Recibido el 4 de enero de 2010, aceptado el 29 de noviembre de 2010

Correspondencia a: Dr. Cristian A. Rojas-Barahona.

Facultad de Educación,

Pontificia Universidad Católica de Chile.

Av. Vicuña Mackenna 4860, Macul, Santiago, Chile.

Se precisa que actualmente el primer autor es académico de esta universidad.

Teléfono: (56 2) 3545362.

E-mail: crojash@uc.cl.

\section{Validación de la prueba de memoria episódica de repetición de la Universidad del Sur de California (USC-REMT)}

\author{
CRISTIAN A. ROJAS-BARAHONA ${ }^{1, \mathrm{a},}$, BEATRIZ ZEGERS PRADO ${ }^{1, \mathrm{a}}$, \\ CARLA E. FÖRSTER MARÍN ${ }^{2, b}$
}

\section{Validation of the University of Southern California Repeatable Episodic Memory Test}

Background: Memory is affected differently with the passage of time; specifically, episodic memory presents a clear decline. There is a relationship of this memory impairment and degenerative and psychiatric diseases. Aim: To determine the reliability and validity of the University of Southern California Repeatable Episodic Memory Test (USC-REMT) in a sample of Chilean adults. Material and Methods: The instrument was administered to 178 subjects living in the Metropolitan Region of Santiago. The sessions were individual, during the morning, lasting approximately 15 minutes. Results: The ANOVA indicates that subjects over 61 years old had lower scores in the test than their younger counterparts. There was an inverse correlation between age and success in "Free recall" ( $r=-0.570)$, "Recognition indicating yes-no " $r=-0.412)$ and "forced-choice recognition" $(r=-0.371)$. Regression analysis showed that age explained $32.1 \%$ "free recall" variance. The three components were grouped into a single factor. Conclusions: The USC-REMT meets the criteria for validity and reliability to be applied for clinical and research purposes.

(Rev Med Chile 2011; 139: 340-347).

Key words: Mental recall; Neurodegenerative diseases; Reproducibility of results.
E xiste consenso en la comunidad científica que la memoria es una habilidad cognitiva que declina con el paso de los años ${ }^{1-7}$. Sin embargo, no se ve igualmente afectada en todas sus dimensiones (por ejemplo, ver 1 y 5 ). La memoria estaría conformada por al menos cinco sistemas diferentes ${ }^{8-9}$ : el procedimental (procesos principalmente implícitos), el de representación perceptiva (o también llamado memoria sensorial), la memoria primaria (sería un símil a la memoria operativa), la memoria semántica y la episódica (en los dos últimos se darían procesos especialmente explícitos). Se confirma la existencia de distintos sistemas de memoria cuando se observa que las tareas que los evalúan, no registran la misma variabilidad con personas que presentan lesiones cerebrales o que simplemente tienen un envejecimiento normal ${ }^{2}$. En los últimos años se ha evidenciando que una de las memorias más afectadas en la senectud es la episódica, observándose un claro declive $e^{5,710}$.

Por memoria episódica (ME) se entiende el sistema que recuerda eventos o experiencias que le han sucedido a la persona en un tiempo y espacio determinado ${ }^{11}$. Existe abundante evidencia científica de la relación del deterioro de la ME y varias enfermedades degenerativas y psiquiátricas. Por ejemplo, representa un síntoma principal en pacientes con la enfermedad de Alzheimer y deterioro cognitivo leve ${ }^{12-13}$; es una dimensión importante a considerar en los trastornos bipolares, en especial en estado eutímico (considerando especialmente el área verbal de la ME ${ }^{14-17}$. En esta línea, el Centro de Trastornos de la Memoria 
(CETRAME), dependiente de la Pontificia Universidad Católica de Chile, identifica entre los diagnósticos más frecuentes el Alzheimer y el deterioro cognitivo leve, además de la depresión ${ }^{18}$. A nivel mundial se observa la misma tendencia, existiendo una proyección de que 65,7 millones de personas estarán afectadas con demencia en el año 2030, cifra que aumentaría a 115,4 millones en el 2050, de los cuales dos tercios serán personas que viven en países de ingresos bajos y medios ${ }^{19}$. Por tanto, un instrumento que evalúe la $\mathrm{ME}$ se hace indispensable, no sólo con fines clínicos, sino también de investigación.

La prueba de Memoria Episódica de Repetición de la Universidad del Sur de California (USC-REMT, por su traducción al inglés) de Parker y $\operatorname{cols}^{20}$, que utiliza contenidos verbales, surge inicialmente para ser sensible a disfunciones del lóbulo frontal y evaluar déficit cognitivos en pacientes con el virus de inmunodeficiencia humana (VIH), donde se presentan un listado de palabras que debían ser recordadas de manera libre ("recuerdo libre"), con el objetivo de evaluar la organización de la información particular o subjetiva del sujeto. Posteriormente, Parker y cols ${ }^{21}$, además de obtener datos normativos en hombres y mujeres, y la aplicación en distintos rangos de edad, incorporaron dos ítems ("reconocimiento sí-no: mayor dependencia del recuerdo" y "reconocimiento obligatorio de tres opciones: mayor dependencia de la familiaridad de los contenidos") buscando sensibilidad de la prueba a distintas formas de memoria. El recuerdo y el reconocimiento difieren en procesos de codificación y recuperación. El primero depende de la iniciación y ejecución de estrategias mnemotécnicas (asociación mental para facilitar el recuerdo) y el segundo, al ser presentado el tema o palabra, reduce la dependencia de estrategias de recuperación más compleja; es decir, el reconocimiento sería más fácil cognitivamente, que el recuerdo, y el recuerdo libre se vería más afectado a los efectos de la edad que el reconocimiento ${ }^{22}$.

La USC-REMT posee una serie de características que la hacen altamente recomendable como una herramienta de detección clínica y de investigación, complementaria a otros instrumentos clásicos, como la prueba de Aprendizaje Verbal de California de Delis y cols ${ }^{23}$. La USC-REMT es una prueba breve (su aplicación dura de 10 a 15 minutos en total), de fácil aplicación, que no necesita de una formación académica avanzada de las personas que la responden, ni tampoco de una capacitación especializada de quien la administra. Al tener siete listas de palabras puede ser aplicada de forma reiterada a los mismos sujetos. Las palabras son simples e independientes de la cultura. Finalmente, se puede usar con adultos de distintas edades, mostrando sensibilidad al deterioro cognitivo asociado con la edad, y a distintos procesos funcionales de la memoria (recuerdo y reconocimiento). Por sus características, ha sido traducida a distintos idiomas, como por ejemplo, francés, griego, vietnamita y español ${ }^{21}$.

El objetivo del presente artículo es analizar la validez y confiabilidad de la prueba USC-REMT en una muestra de adultos de distintas edades (20 a 95 años) para su aplicación en el campo clínico e investigación.

\section{Material y Métodos}

La muestra estuvo conformada por 178 personas adultas (67 hombres y 111 mujeres) de diferentes organizaciones sociales urbanas de la Región Metropolitana de Santiago, Chile, las cuales fueron seleccionadas mediante un muestreo intencionado por cuotas, considerando como criterios de selección la edad y el sexo. Se excluyeron las personas que: tenían menos de 8 años de escolaridad (con el fin de asegurar un desarrollo de habilidades cognitivas básicas ${ }^{24}$ ), y que reportaron no estar "sanos". Siguiendo la clasificación del estudio de Parker y cols $^{21}$, se conformaron tres grupos, Grupo 1: 54 sujetos ( 25 hombres y 29 mujeres) con una edad que fluctuaba entre 20 y 40 años; Grupo 2: 49 sujetos (19 hombres y 30 mujeres) con una edad entre 41 y 60 años; y Grupo 3: 75 sujetos (23 hombres y 52 mujeres) que tenían 61 años o más. Las medias de edad de los tres grupos fueron 30,95 años, 52,4 años y 74 años, respectivamente. El detalle por edad y sexo se presenta en la Tabla 1.

\section{Descripción del instrumento y procedimiento de aplicación}

La USC-REMT desarrollada por Parker y cols $^{20-21}$ consta de 3 partes, en cada una la persona debe utilizar una estrategia distinta para recordar o reconocer ("recuerdo libre", "reconocimiento indicando sí o no" y "reconocimiento de elección forzada") las palabras de una lista de 15 conteni- 
Tabla 1. Distribución de la muestra según grupo, sexo y edad

\begin{tabular}{|cllcccc|}
\hline Grupo & Sexo & $\mathbf{n}$ & Media & DS & Mínimo & Máximo \\
\hline $\begin{array}{c}1 \\
(20-40 \text { años })\end{array}$ & Mujer & 29 & 31,11 & 7,09 & 20 & 40 \\
& Hombre & 25 & 30,75 & 6,22 & 20 & 39 \\
& Total & 54 & 30,95 & 6,64 & 20 & 40 \\
$(41-60$ años $)$ & Mujer & 30 & 51,42 & 5,92 & 41 & 60 \\
& Hombre & 19 & 53,95 & 5,63 & 42 & 60 \\
$(>61$ años $)$ & Total & 49 & 52,40 & 5,88 & 41 & 60 \\
& Mujer & 52 & 76,16 & 9,31 & 61 & 95 \\
& Hotal & 23 & 69,11 & 7,01 & 61 & 89 \\
\hline
\end{tabular}

dos semánticos no relacionados (sustantivos de uso común). Los autores presentan siete listas de palabras distintas, consideradas versiones paralelas de la prueba. Las instrucciones y las palabras fueron traducidas desde el inglés al español y evaluadas por 5 jueces expertos, para asegurar el sentido original y la pertinencia al léxico chileno, estableciendo como criterio de aceptación 100\% de concordancia entre ellos.

Previo a la aplicación, a cada participante se le explicó el objetivo de la prueba y sus características, señalándoles que era de carácter voluntario, que no tendría consecuencias adversas para su salud y que no recibirían ningún pago por su participación. Se les comunicó que la información sería utilizada de manera colectiva, con fines de investigación, y que podían abandonar el test si ellos lo estimaban conveniente. Los participantes respondieron el test en forma individual y se aplicó 1 lista por sujeto en forma aleatoria.

En la primera parte del test ("Recuerdo libre") se leyó una lista de 15 palabras (lista inicial) y la persona debió señalar el mayor número de palabras que recordaba. El procedimiento se repitió 3 veces y se registró el número de palabras dichas por el sujeto, clasificándolas en: correctas, repetidas e inventadas. La segunda parte consistió en la lectura, una a una, de 45 palabras frente a las cuales la persona debió señalar si correspondía o no al listado inicial ("Reconocimiento indicando sí-no"), registrándose el número de éxitos y fracasos (falsas alarmas). En la tercera, se leyó tríos de palabras donde el sujeto debió señalar cuál de las tres correspondía al listado inicial ("Reconocimiento de elección forzada"), anotando el número de palabras correctamente reconocidas. La duración de la aplicación fue de 15 minutos aproximadamente y se realizó antes del medio día. El instrumento completo, con el listado de palabras y las instrucciones en español, se puede solicitar a los autores del artículo.

\section{Resultados}

En la Tabla 2 se presentan los estadísticos descriptivos de las medidas de memoria de los sujetos, segmentadas por edad. El total de casos válidos para todos los análisis fue $\mathrm{n}=178$. Para la medición "Recuerdo libre", el máximo obtenido fue de 35 palabras recordadas de un total de 45 , mientras que para las dos medidas de reconocimiento (Reconocimiento indicando sí-no/Reconocimiento de elección forzada) se alcanzó el máximo teórico de 15 palabras.

El análisis de varianza (ANOVA) indica que en "Recuerdo libre", no hubo diferencias entre sexo $\left(\mathrm{F}_{(178,1)}=0,348 ; \mathrm{p}=0,556\right)$ ni efecto de interacción sexo-edad $\left(\mathrm{F}_{(178,2)}=0,583 ; \mathrm{p}=0,559\right)$, pero si hubo diferencias de medias entre los tres grupos de edad $\left(\mathrm{F}_{(178,2)}=27,843 ; \mathrm{p}=0,000\right)$, donde el número de palabras recordadas disminuyó paulatinamente a medida que la edad aumentaba. Respecto del "Reconocimiento indicando sí-no", no hubo di- 
ARTÍCULO DE INVESTIGACIÓN

Prueba de Memoria Episódica de Repetición USC - C. A. Rojas-Barahona et al

Tabla 2: Estadísticos descriptivos de los puntajes de las medidas de memoria

\begin{tabular}{|c|c|c|c|c|c|c|c|}
\hline Sección & Edad (años) & Sexo & $\mathbf{n}$ & Media & DS & Mínimo & Máximo \\
\hline \multirow[t]{9}{*}{ Recuerdo libre } & \multirow[t]{3}{*}{$20-40$} & Mujer & 29 & 26 & 6 & 11 & 35 \\
\hline & & Hombre & 25 & 24 & 3 & 18 & 31 \\
\hline & & Total & 54 & 25 & 5 & 11 & 35 \\
\hline & \multirow[t]{3}{*}{$41-60$} & Mujer & 30 & 21 & 5 & 11 & 32 \\
\hline & & Hombre & 19 & 21 & 6 & 12 & 33 \\
\hline & & Total & 49 & 21 & 5 & 11 & 33 \\
\hline & \multirow[t]{3}{*}{$>61$} & Mujer & 52 & 18 & 5 & 8 & 29 \\
\hline & & Hombre & 23 & 18 & 4 & 10 & 23 \\
\hline & & Total & 75 & 18 & 5 & 8 & 29 \\
\hline \multirow[t]{9}{*}{ Reconocimiento si/no } & \multirow[t]{3}{*}{$20-40$} & Mujer & 29 & 14 & 1 & 12 & 15 \\
\hline & & Hombre & 25 & 14 & 1 & 12 & 15 \\
\hline & & Total & 54 & 14 & 1 & 12 & 15 \\
\hline & \multirow[t]{3}{*}{$41-60$} & Mujer & 30 & 14 & 1 & 11 & 15 \\
\hline & & Hombre & 19 & 13 & 2 & 8 & 15 \\
\hline & & Total & 49 & 14 & 2 & 8 & 15 \\
\hline & \multirow[t]{3}{*}{$>61$} & Mujer & 52 & 13 & 3 & 3 & 15 \\
\hline & & Hombre & 23 & 13 & 3 & 5 & 15 \\
\hline & & Total & 75 & 13 & 3 & 3 & 15 \\
\hline \multirow[t]{9}{*}{ Reconocimiento falsas alarmas } & \multirow[t]{3}{*}{$20-40$} & Mujer & 29 & 1 & 1 & 0 & 4 \\
\hline & & Hombre & 25 & 2 & 4 & 0 & 20 \\
\hline & & Total & 54 & 1 & 3 & 0 & 20 \\
\hline & \multirow[t]{3}{*}{$41-60$} & Mujer & 30 & 2 & 4 & 0 & 18 \\
\hline & & Hombre & 19 & 1 & 2 & 0 & 9 \\
\hline & & Total & 49 & 2 & 3 & 0 & 18 \\
\hline & \multirow[t]{3}{*}{$>61$} & Mujer & 52 & 2 & 3 & 0 & 19 \\
\hline & & Hombre & 23 & 3 & 3 & 0 & 8 \\
\hline & & Total & 75 & 2 & 3 & 0 & 19 \\
\hline \multirow[t]{9}{*}{ Reconocimiento elección forzada } & \multirow[t]{3}{*}{$20-40$} & Mujer & 29 & 14 & 2 & 6 & 15 \\
\hline & & Hombre & 25 & 15 & 1 & 9 & 15 \\
\hline & & Total & 54 & 14 & 2 & 6 & 15 \\
\hline & \multirow[t]{3}{*}{$41-60$} & Mujer & 30 & 14 & 1 & 11 & 15 \\
\hline & & Hombre & 19 & 13 & 3 & 4 & 15 \\
\hline & & Total & 49 & 14 & 2 & 4 & 15 \\
\hline & \multirow[t]{3}{*}{$>61$} & Mujer & 52 & 13 & 2 & 7 & 15 \\
\hline & & Hombre & 23 & 12 & 4 & 1 & 15 \\
\hline & & Total & 75 & 12 & 3 & 1 & 15 \\
\hline
\end{tabular}


ferencias por sexo $\left(\mathrm{F}_{(178,1)}=0,484 ; \mathrm{p}=0,487\right) \mathrm{ni}$ efecto de interacción sexo-edad $\left(\mathrm{F}_{(178,2)}=0,506 ; \mathrm{p}\right.$ $=0,604)$, sólo hubo diferencias entre las medias de los grupos de edad $\left(\mathrm{F}_{(178,2)}=10,956 ; \mathrm{p}=0,000\right)$, específicamente entre el grupo $>61$ y los otros dos. En las falsas alarmas no hubo diferencias por sexo $\left(\mathrm{F}_{(178,1)}=0,799 ; \mathrm{p}=0,373\right)$, por edad $\left(\mathrm{F}_{(178,2)}=1,848\right.$; $\mathrm{p}=0,161)$ ni efectos de interacción $\left(\mathrm{F}_{(178,2)}=1,698\right.$; $\mathrm{p}=0,186)$. En la medición de "Reconocimiento de elección forzada", no hubo diferencias por sexo $\left(\mathrm{F}_{(178,1)}=3,375 ; \mathrm{p}=0,068\right)$ ni efecto de interacción sexo-edad $\left(\mathrm{F}_{(178,2)}=1,543 ; \mathrm{p}=0,217\right)$. Las diferencias entre los grupos de edad $\left(\mathrm{F}_{(178,2)}=13,031\right.$; $p=0,000)$ se dieron entre $>61$ y los otros dos.

El ANOVA realizado con las listas de palabras utilizadas, indica que no hubo diferencias en "Recuerdo libre" $\left(\mathrm{F}_{(178,6)}=640 ; \mathrm{p}=0,698\right)$, "Reconocimiento indicando sí-no" $\left(\mathrm{F}_{(178,6)}=1,254 ; \mathrm{p}=\right.$ $0,281)$ ni en "Reconocimiento de elección forzada" $\left(\mathrm{F}_{(178,6)}=1,123 ; \mathrm{p}=0,351\right)$, lo que indica que las siete listas son formas paralelas de la prueba y que los resultados de ME serían válidos independiente de la lista que se utilice.

El análisis de Correlación de Pearson mostró una relación inversa entre la edad y el éxito en "Recuerdo libre" ( $r=-0,570)$, "Reconocimiento indicando sí-no" $(r=-0,412)$ y "Reconocimiento de elección forzada" $(r=-0,371)$. También se observó una relación directa entre las tres medidas de memoria y una relación inversa entre éstas y el reconocimiento de falsas alarmas (Tabla 3 ). Todas las medidas de memoria correlacionaron directa y significativamente con el puntaje total de la prueba (valores superiores a 0,7 ), por lo tanto, la consistencia interna entre el "ítem" y el test tuvo índices considerados buenos. El índice alfa de Crombach con los tres ítems fue de 0,646. El análisis de Regresión hecho con cada medida de memoria como variable dependiente y la edad como variable predictora señalan que para "Recuerdo libre" la edad explicó 32,1\%, para "Reconocimiento indicando sí-no" $16,5 \%$ y para "Reconocimiento de elección forzada" $13,3 \%$.

El análisis por percentiles para los tres grupos de edad evidenció que a mayor edad, menor fue el número de respuestas correctas en cada medida de memoria (Tabla 4). No se consideró la diferenciación por sexo, dado que los análisis señalan que no hay diferencias entre estos grupos.

El análisis Factorial Exploratorio de Componentes Principales mostró que las tres partes de la prueba se agrupan en un factor que explica el $68,2 \%$ de la varianza total (Tabla 5), y los pesos factoriales fueron superiores a 0,81 (Tabla 6), lo que indica que las tres partes miden el mismo constructo, que corresponde a la ME. No se consideró la rotación de los componentes porque los datos originales eran lo suficientemente claros y los análisis posteriores no aportan nueva información.

Tabla 3. Correlaciones entre edad y medidas de memoria

\begin{tabular}{|c|c|c|c|c|c|}
\hline & $\begin{array}{l}\text { Recuerdo } \\
\text { libre }\end{array}$ & $\begin{array}{c}\text { Reconocimiento } \\
\text { Sí - No }\end{array}$ & $\begin{array}{l}\text { Reconocimiento } \\
\text { falsas alarmas }\end{array}$ & $\begin{array}{l}\text { Reconocimiento } \\
\text { elección forzada }\end{array}$ & $\begin{array}{c}\text { Puntaje } \\
\text { total }\end{array}$ \\
\hline Edad & $-0,570$ * & $-0,412^{*}$ & 0,133 & $-0,371$ * & $-0,576^{*}$ \\
\hline Recuerdo libre & & $0,538^{*}$ & $-0,300^{*}$ & $0,542^{*}$ & $0,937^{*}$ \\
\hline $\begin{array}{l}\text { Reconocimiento } \\
\text { Sí-No }\end{array}$ & & & $-0,004$ & $0,489 *$ & $0,722 *$ \\
\hline $\begin{array}{l}\text { Reconocimiento } \\
\text { falsas alarmas }\end{array}$ & & & & $-0,315^{*}$ & $-0,290$ * \\
\hline $\begin{array}{l}\text { Reconocimiento } \\
\text { elección forzada }\end{array}$ & & & & & $0,757^{*}$ \\
\hline
\end{tabular}

* La correlación es significativa al nivel 0,01 (bilateral). 
Prueba de Memoria Episódica de Repetición USC - C. A. Rojas-Barahona et al

Tabla 4. Distribución en percentiles del puntaje de cada medida según edad

\begin{tabular}{|c|c|c|c|c|c|c|c|c|c|c|c|c|}
\hline \multirow{2}{*}{$\begin{array}{l}\text { Edad } \\
\text { (años) }\end{array}$} & \multicolumn{3}{|c|}{ Recuerdo libre } & \multicolumn{3}{|c|}{$\begin{array}{c}\text { Reconocimiento } \\
\text { sí/no }\end{array}$} & \multicolumn{3}{|c|}{$\begin{array}{l}\text { Reconocimiento } \\
\text { falsas alarmas }\end{array}$} & \multicolumn{3}{|c|}{$\begin{array}{l}\text { Reconocimiento } \\
\text { elección forzada }\end{array}$} \\
\hline & \multirow[t]{2}{*}{$20-40$} & \multirow[t]{2}{*}{$41-60$} & \multirow[t]{2}{*}{$>61$} & \multirow[t]{2}{*}{$20-40$} & \multirow[t]{2}{*}{$41-60$} & \multirow[t]{2}{*}{$>61$} & \multirow[t]{2}{*}{$20-40$} & \multirow[t]{2}{*}{$41-60$} & \multirow[t]{2}{*}{$>61$} & \multirow[t]{2}{*}{$20-40$} & \multirow[t]{2}{*}{$41-60$} & \multirow[t]{2}{*}{$>61$} \\
\hline Percentiles & & & & & & & & & & & & \\
\hline 1 & 11 & 11 & 8 & 12 & 8 & 3 & 0 & 0 & 0 & 6 & 4 & 1 \\
\hline 5 & 16 & 12 & 8 & 12 & 10 & 7 & 0 & 0 & 0 & 10 & 8 & 6 \\
\hline 10 & 18 & 13 & 11 & 13 & 12 & 9 & 0 & 0 & 0 & 13 & 11 & 9 \\
\hline 20 & 20 & 16 & 13 & 14 & 13 & 11 & 0 & 0 & 0 & 14 & 12 & 10 \\
\hline 30 & 22 & 19 & 16 & 14 & 13 & 12 & 0 & 0 & 0 & 15 & 14 & 11 \\
\hline 40 & 23 & 20 & 17 & 14 & 14 & 12 & 0 & 0 & 0 & 15 & 14 & 12 \\
\hline 50 & 25 & 21 & 18 & 15 & 15 & 13 & 0 & 1 & 1 & 15 & 15 & 13 \\
\hline 60 & 26 & 23 & 19 & 15 & 15 & 14 & 1 & 1 & 2 & 15 & 15 & 14 \\
\hline 70 & 28 & 24 & 20 & 15 & 15 & 14 & 1 & 1 & 2 & 15 & 15 & 15 \\
\hline 80 & 30 & 25 & 22 & 15 & 15 & 15 & 1 & 3 & 4 & 15 & 15 & 15 \\
\hline 90 & 33 & 28 & 23 & 15 & 15 & 15 & 4 & 5 & 6 & 15 & 15 & 15 \\
\hline 95 & 33 & 32 & 26 & 15 & 15 & 15 & 5 & 9 & 7 & 15 & 15 & 15 \\
\hline 99 & 35 & 33 & 29 & 15 & 15 & 15 & 20 & 18 & 19 & 15 & 15 & 15 \\
\hline
\end{tabular}

Tabla 5. Análisis factorial de componentes principales y varianza total explicada

\begin{tabular}{|ccccccc|}
\hline Componente & \multicolumn{3}{c}{ Autovalores iniciales } & \multicolumn{2}{c|}{$\begin{array}{c}\text { Sumas de las saturaciones } \\
\text { al cuadrado de la extracción }\end{array}$} \\
& Total & \% varianza & \% acumulado & Total & \% varianza & \% acumulado \\
\hline 1 & 2,046 & 68,207 & 68,207 & 2,046 & 68,207 & 68,207 \\
\hline 3 & 0,511 & 17,027 & 85,234 & & & \\
\hline
\end{tabular}

Tabla 6. Matriz de componentes y pesos factoriales de las tres partes de la prueba

\begin{tabular}{|lc|}
\hline Sección & Componente 1 \\
Recuerdo libre & 0,843 \\
Reconocimiento Sí-No & 0,816 \\
Reconocimiento elección forzada & 0,818 \\
\hline
\end{tabular}

Método de extracción: Análisis de componentes principales. 1 componente extraído.

\section{Discusión}

En términos de la validez, las correlaciones entre las tres partes de la prueba muestran que hay una relación estrecha entre ellas, lo que sumado a la agrupación en un sólo factor indicaría que la prueba mide la ME como constructo.

En cuanto a la confiabilidad de la prueba, el índice Alfa de Crombach es de un valor aceptable $(0,646)$ considerando el número de ítems, sin embargo, los resultados de la correlación ítem- 
test evidencian que la prueba cuenta con una consistencia interna considerada buena $\left(\mathrm{r}_{\text {recuerdo libre }}\right.$ $\left.=0,937 ; r_{\text {recon. si-no }}=0,722 ; r_{\text {recon. elección forzada }}=0,735\right)$, por tanto, se puede afirmar que la USC-REMT, es un instrumento confiable.

Dado que no se observaron diferencias entre las listas de palabras, se puede considerar que los listados constituyen versiones paralelas del test, lo que conlleva ventajas para la aplicación en reiteradas oportunidades, sin tener como factor de invalidez el aprendizaje o recuerdo de los ítems.

Por otra parte, la ausencia de diferencias por sexo indica que la prueba no tendría sesgo de esta variable para la muestra de este estudio, al igual como reportan Parker y cols ${ }^{21}$. Sin embargo, hay que ser prudente, porque existen investigaciones que han observado distintos desempeños de la $\mathrm{ME}$ en hombres y mujeres, aunque utilizando otros instrumentos de medición ${ }^{25}$. En dichas investigaciones se ha resaltado el papel de la cultura.

Respecto de las puntuaciones máximas de cada parte de la prueba, es posible señalar que en "Recuerdo libre", el máximo obtenido fue de 35 respuestas correctas de un total de 45 en el grupo más joven (20-40 años), lo que señalaría que esta medida no tendría "techo" para quienes respon$\mathrm{den}^{21}$. Aunque en las mediciones de reconocimiento se alcanzan los puntajes máximos, el análisis por percentiles muestra que en los grupos de mayor edad, el máximo se alcanza en el $\mathrm{P}_{80}$ para "Reconocimiento indicando sí-no" y en el $\mathrm{P}_{70}$ en "Reconocimiento de elección forzada", lo que indicaría que la parte de "reconocimiento" de la prueba es de mayor utilidad para establecer diagnósticos diferenciados o para continuar estudiando el comportamiento de la memoria con el paso de los años, especialmente en el grupo de 61 años en adelante.

Las diferencias entre los grupos de edad son esperables y coherentes con los resultados observados por Parker y cols ${ }^{21}$. Respecto de la relación entre la edad y la ME, los resultados muestran que hay una correlación inversa entre ellas; es decir, a mayor edad, menor es la capacidad de memoria de las personas. Así, el análisis de la capacidad predictiva de la edad sobre los distintos procesos funcionales de la memoria, indica que "Recuerdo libre" presenta mayor porcentaje de varianza explicada $\left(\mathrm{R}^{2}=0,32\right)$, seguido de "Reconocimiento indicando sí-no" $\left(\mathrm{R}^{2}=0,16\right)$ y finalmente "Reconocimiento de elección forzada" $\left(\mathrm{R}^{2}=0,13\right)$. Aunque los porcentajes de varianza de las medidas de reconocimiento no son sustantivos, la aplicación de estos ítems entrega información acerca de los procesos funcionales de la memoria (recuerdo y reconocimiento).

Se hace necesario considerar en la aplicación de futuras pruebas de memoria u otras que evalúen el desempeño cognitivo de adultos, especialmente mayores, el momento del día. Se ha documentado, en estudios recientes, que en aplicaciones realizadas al medio día, los resultados de las pruebas de memoria decrecen significativamente ${ }^{26}$.

Como limitaciones del estudio se podrían mencionar la muestra reducida, sin embargo, los resultados indican un comportamiento similar a lo observado en la revisión original, con tamaños muestrales de las mismas dimensiones (se consideró 112 personas entre 18 y 93 años $^{21}$ ), y la no utilización de pruebas de tamizaje para certificar posibles deterioros cognitivos no reportados.

Se puede concluir que la USC-REMT es una prueba adecuada para ser aplicada a sujetos adultos en Chile, tanto para fines clínicos como de investigación. El uso clínico de la prueba podría facilitar, en conjunto con otras pruebas (como por ejemplo, el instrumento de tamizaje compuesto por el Mini-Mental State Examination y el test de Actividades Funcionales de Pfeffer ${ }^{27}$ ), diagnósticos tempranos de patologías específicas como deterioros cognitivos leves, Alzheimer, el efecto del uso de drogas, o para detectar factores propios de la senectud. Su uso en investigación sería un aporte, no tan sólo en estudios transversales, sino también en longitudinales, especialmente por su característica de uso repetido (cuenta con 7 listas). Por todos los antecedentes mencionados, además de su fácil aplicación y bajo costo, la prueba USCREMT puede ser un aporte significativo en un área de la salud poco explorada y con un evidente aumento de la población de impacto ${ }^{26-28}$, como es la especialidad médica de geriatría, además de otras especialidades, como la psiquiatría y la neurología. Al mismo tiempo sería de utilidad a otros profesionales de la salud, como psicólogos.

Es importante destacar que la presente versión de la USC-REMT es la primera publicación realizada en Chile, y aun cuando hay antecedentes que señalan la traducción al español ${ }^{21}$, hasta donde se ha podido conocer, no hay artículos científicos en dicha lengua, permitiendo conformarse en una base para futuras adaptaciones y estudios normativos para países de habla hispana. 
Agradecimientos. De manera especial agradecemos el apoyo y disposición de la autora de la prueba USC-REMT Elizabeth S. Parker, quien nos facilitó el material, autorizó su uso y resolvió dudas.

\section{Referencias}

1. Balota DA, Dolan PO, Ducheck JM. Memory changes in healthy older adults. En Tulving E, Craik FIM, editores, The Oxford handbook of memory. New York: Oxford University Press; 2000; 395-409.

2. Craik FMI. Cambios en la memoria humana relacionados con la edad. En Park D, Schwarz N, editores, Envejecimiento cognitivo. Madrid: Panamericana; 2002; 77-93.

3. Jonker C, Geerlings MI, Schmand B. Are memory complaints predictive for dementia? A review of clinical and population based studies. Int J Psychiatry 2000; 15: 98391.

4. Luo L, Craik FIM. Aging and memory: A cognitive approach. Can J Psychiatry 2008; 53: 346-53.

5. Nilsson LG. Memory functioning in normal aging. Acta Neurol Scand 2003; 107: 7-13.

6. Zacks RT, Hasher L. Aging and long-term memory: Deficits are not inevitable. En Bialystok E., Craik FIM, editores, Lifespan Cognition. United States of America: Oxford University Press; 2006; 162-77.

7. Zacks RT, Hasher L, Li KZH. Human memory. En Craik FIM, Salthouse TA, editores, The handbook of aging and cognition. Mahwah, NJ: Erlbaum; 1999; 293-357.

8. Tulving E, Schacter DL. Priming and human memory systems. Science 1990; 247: 301-6.

9. Schacter DL, Tulving E. What are the memory systems of 1994? En Schacter DL, Tulving E, editores, Memory Systems. Cambridge, MA: MIT Press; 1994; 1-38.

10. Siedlecki KL. Investigating the structure and age invariance of episodic memory across the adult lifespan. Psychol Aging 2007; 22: 251-68.

11. Tulving E. Episodic and semantic memory. En Tulving E, Donaldson W, editors, Organization of memory. New York: Academic Press; 1972; 381-403.

12. Collie A, Maruff P. The neuropsychology of preclinical Alzheimer's disease and mild cognitive impairment. Neurosci Biobehav Rev 2000; 24: 365-74.

13. Bennett IJ, Golob EJ, Parker ES, Starr AA. Memory evaluation in mild cognitive impairment using recall and recognition tests. J Clin Exp Neuropsychol 2006; 28: 1408-22.

14. Graf P, Schacter DL. Implicit and explicit memory for new associations in normal and amnestic subjects. J Exp Psychol Learn 1985; 13: 45-53.

15. Van Gorp WG, Altshuler L, Theberge DC, Mintz J. Declarative and procedural memory in bipolar disorder. Biol Psychiatry 1999; 46: 525-531.

16. Martínez-Arán A, Vieta E, Colom F, Torrent C, Sánchez-Moreno J, Reinares M. Cognitive impairment in euthymic bipolar patients: implications for clinical and functional outcome. Bipolar Disord 2002; 6: 224-32.

17. Balanza-Martínez V, Tabares-Seisdedos R, Selva-Vera G, Martínez-Arán A, Torrent C, Salazar-Fraile J. Persistent cognitive dysfunctions in bipolar I disorder and schizophrenic patients: a 3 year follow-up study. Psychother Psychosom 2005; 74: 113-9.

18. González J, Galdames D, Oporto S. Perfiles diagnósticos y epidemiológicos en una Unidad de Memoria. Rev Med Chile 2005; 133: 789-94.

19. Wimo A, Prince M. World Alzheimer Report 2010. The Global Economic Impact of Dementia. London, UK: Alzheimer's Disease International; 2010.

20. Parker ES, Eaton EM, Whipple SC, Hesertine PNR, Bridge TP. University of Southern California Repeatable Episodic Memory Test. J Clin Exp Neuropsychol 1995; 17: 926-36.

21. Parker ES, Landau SM, Whipple SC, Schwartz BL. Aging, recall and recognition: A study on the sensitivity of the University of Southern California Repeatable Episodic Memory Test (USC-REMT). J Clin Exp Neuropsychol 2004; 26: 428-40.

22. Craik FIM, Mcdowd JM. Age differences in recall and recognition. J Exp Psychol Learn Mem Cogn 1987; 13: 474-9.

23. Delis DC, Kramer JH, Kaplan E, Ober BA. The California Verbal Learning Test. New York: The Psychological Corporation; 1987.

24. Gallart MA, Jacinto C. Competencias laborales: Tema clave en la articulación Educación-Trabajo. Bol Red Latin Educ Trab 1995; 6: 59-62.

25. Herlitz A, Rehnman J. Sex differences in episodic memory. Curr Dir Psychol Sci 2008; 17: 52-6.

26. Martin B, Buffngton ALH, Welsh-Bohmer KA, Brandt J. Time of day affects episodic memory in older adults. Aging Neuropsychol Cogn 2008; 15: 146-64.

27. Quiroga P, Albala C, Klaasen G. Validación de un test de tamizaje para el diagnóstico de demencia asociada a edad, en Chile. Rev Med Chile 2004; 132: 467-78.

28. Marín PP, Guzmán JM, Araya A. Adultos mayores institucionalizados en Chile: ¿Cómo saber cuántos son? Rev Med Chile 2004; 132: 832-8. 